\title{
Research
}

\section{Delivery and impact of the NHS Health Check in the first 8 years:}

\author{
a systematic review
}

\begin{abstract}
Background

Since 2009, all eligible persons in England have been entitled to an NHS Health Check. Uncertainty remains about who attends, and the health-related impacts.

\section{Aim}

To review quantitative evidence on coverage (the proportion of eligible individuals who attend), uptake (proportion of invitees who attend), and impact of NHS Health Checks.

\section{Design and setting}

A systematic review and quantitative data synthesis. Included were studies or data reporting coverage or uptake and studies reporting any health-related impact that used an appropriate comparison group or beforeand-after study design.
\end{abstract}

\section{Method}

Eleven databases and additional internet sources were searched to November 2016 .

\section{Results}

Twenty-six observational studies and one additional dataset were included. Since 2013, $45.6 \%$ of eligible individuals have received health check. Coverage is higher among older people, those with a family history of coronary heart disease, those living in the most deprived areas, and some ethnic minority groups. Just under half (48.2\%) of those invited have taken up the invitation. Data on uptake and impact (especially regarding health-related behaviours) are limited. Uptake is higher in older people and females, but lower in those living in the most deprived areas. Attendance is associated with small increases in disease detection, decreases in modelled cardiovascular disease risk, and increased statin and antihypertensive prescribing.

\section{Conclusion}

Published attendance, uptake, and prescribing rates are all lower than originally anticipated, and data on impact are limited, with very few studies reporting the effect of attendance on health-related behaviours. High-quality studies comparing matched attendees and non-attendees and health economic analyses are required.

\section{Keywords}

coverage; general practice; impact; NHS Health Check; primary care; systematic review; uptake.

\section{INTRODUCTION}

The NHS Health Check programme was launched in England in 2009 as part of a healthcare strategy aimed at empowering patients and preventing illness." It offers everyone aged $40-74$ years without preexisting cardiovascular disease (CVD), chronic kidney disease (CKD), type 2 diabetes (T2DM), or dementia an assessment of their risk of having or developing such conditions, and advice about relevant medications and lifestyle changes every 5 years. Since 2013, local authorities have had a statutory responsibility to offer the programme to all eligible individuals, with funding provided by Public Health England (PHE). ${ }^{2}$ Echoing similar efforts in other countries to provide preventive health checks, ${ }^{3,4}$ the programme is delivered by various providers, predominantly general practices.

The programme was introduced simultaneously nationwide without robust economic evaluation evidence from a randomised controlled trial (RCT), and with very limited available evidence on health check strategies implemented in other countries. ${ }^{5,6}$ However, the Department of Health modelled the potential long-term cost-effectiveness of the programme.? In that modelling, it was envisaged that all

A Martin, PhD, research fellow, Academic Unit of Health Economics, Leeds Institute of Health Sciences, University of Leeds, Leeds, and RAND Europe, Cambridge. CL Saunders, PhD, senior research associate, Primary Care Unit, Department of Public Health and Primary Care, University of Cambridge, and RAND Europe Cambridge. E Harte, MSc, analyst; C MacLure, MA, analyst, RAND Europe, Cambridge. J Mant MD, professor; FM Walter, MD, principal researcher; JA Usher-Smith, PhD, clinical senior research associate, Primary Care Unit, Department of Public Health and Primary Care University of Cambridge, Cambridge. SJ Griffin DM, professor, Primary Care Unit, Department of Public Health and Primary Care, and Medical Research Council (MRC) Epidemiology Unit, Institute of Metabolic Science, University of those eligible would be invited for an NHS Health Check during the first 5-year cycle. Based on evidence from a national breast screening programme, it was expected that $75 \%$ would attend. ${ }^{7}$ Of those attendees with high cholesterol or CVD risk (10-year risk $\geq 20 \%$ ), it was hoped that $85 \%$ would be prescribed statins (in 50\% of cases, this was attributed directly to the health check). Using a time horizon of a lifetime, the cost-effectiveness of the programme was predicted in this modelling to be $€ 2866$ per quality adjusted life year (QALY) (20152016 prices), ${ }^{8}$ well within the limit of what would normally be deemed cost-effective by the National Institute for Health and Care Excellence.

In order to provide up-to-date estimates of delivery and impact of the NHS Health Check, the objectives of this study were to systematically identify and synthesise available evidence on:

- coverage (the proportion of the eligible population who have attended an NHS Health Checkl and variation in coverage;

- uptake (the proportion of those invited who have attended an NHS Health Check) and variation in uptake; and

- the effect of the programme.

Cambridge, Cambridge. C Meads, PhD, professor RAND Europe, and Faculty of Health, Social Care and Education, Anglia Ruskin University, Cambridge.

\section{Address for correspondence}

Adam Martin, Academic Unit of Health Economics, Leeds Institute of Health Sciences, University of Leeds, Leeds LS2 9LJ, UK.

Email: A.Martin1aleeds.ac.uk

Submitted: 9 October 2017; Editor's response: 11 December 2017; final acceptance: 14 February 2018.

OBritish Journal of General Practice

This is the full-length article (published online 19 Jun 2018) of an abridged version published in print. Cite this version as: Br J Gen Pract 2018; DOI: https://doi.org/10.3399/bjgp18X697649 


\section{How this fits in}

Simultaneous nationwide rollout in 2009 of the NHS Health Check programme was based on some strong assumptions about the likely impact of the programme. Almost a decade on, there remains much uncertainty about who attends and the overall health benefits. This is the first systematic review of quantitative data from the programme. Although the authors found attendance is much lower than originally anticipated, attendees cannot be readily characterised as the 'worried well' or 'easiest to reach".

\section{METHOD}

\section{Search strategy and study selection}

Full details of the search strategy are the study selection process is described in detail elsewhere. ${ }^{10}$ Searches included 11 literature databases and additional internet sources encompassing both peer-reviewed and grey literature relevant to NHS Health Checks, published up to November 2016.

\section{Inclusion criteria}

Quantitative observational data or analyses (cross-sectional or longitudinal) that included people eligible for an NHS Health Check and reported evidence on coverage or uptake were included. Impact studies reporting any health-related outcome that used an appropriate comparison group or a before-and-after study design were also included. Data or analyses relating to other screening or health check services that were not NHS Health Checks were excluded, as were editorials and opinion pieces.

\section{Data extraction, quality assessment, and} synthesis

Data were extracted independently by three researchers using forms devised for this study. Reflecting the wide range of study designs, data, and methods identified, existing Critical Appraisal Skills Programme (CASP) checklists ${ }^{11}$ were adapted for the quality assessment of identified studies.

For each objective, the authors grouped studies according to their design. As the programme runs in 5-year cycles, where necessary, the authors adjusted reported coverage to a standardised measure of coverage per year per one-fifth of the total eligible population (which can lead to coverage exceeding $100 \%$ if more than $20 \%$ of the eligible population attend in a given year). The authors categorised the health-related impact studies into four groups (disease detection, health related behaviours, prescribing, and individual risk factors), and report the results in order of the degree to which observed differences between groups can be attributed to NHS Health Check attendance.

\section{RESULTS}

\section{Overview of included studies}

The searches identified 18524 articles. The authors reviewed 178 full-text articles, and 26 (including five from the grey literature) ${ }^{12-16}$ were deemed relevant (Figure 1). All were observational studies. Seven used data from large, routine, consolidated datasets with nationwide reach ${ }^{15,17-22}$ (including the Clinical Practice Research Datalink [CPRD, ${ }_{1}^{17-20}$ QResearch, $^{21}$ and prescribing data): ${ }^{15} 19$ used local data from general practices $(n=17)^{13,14,23-37}$ or community settings $(n=2)$ 


\section{Table 1. NHS Health Check: overall coverage}

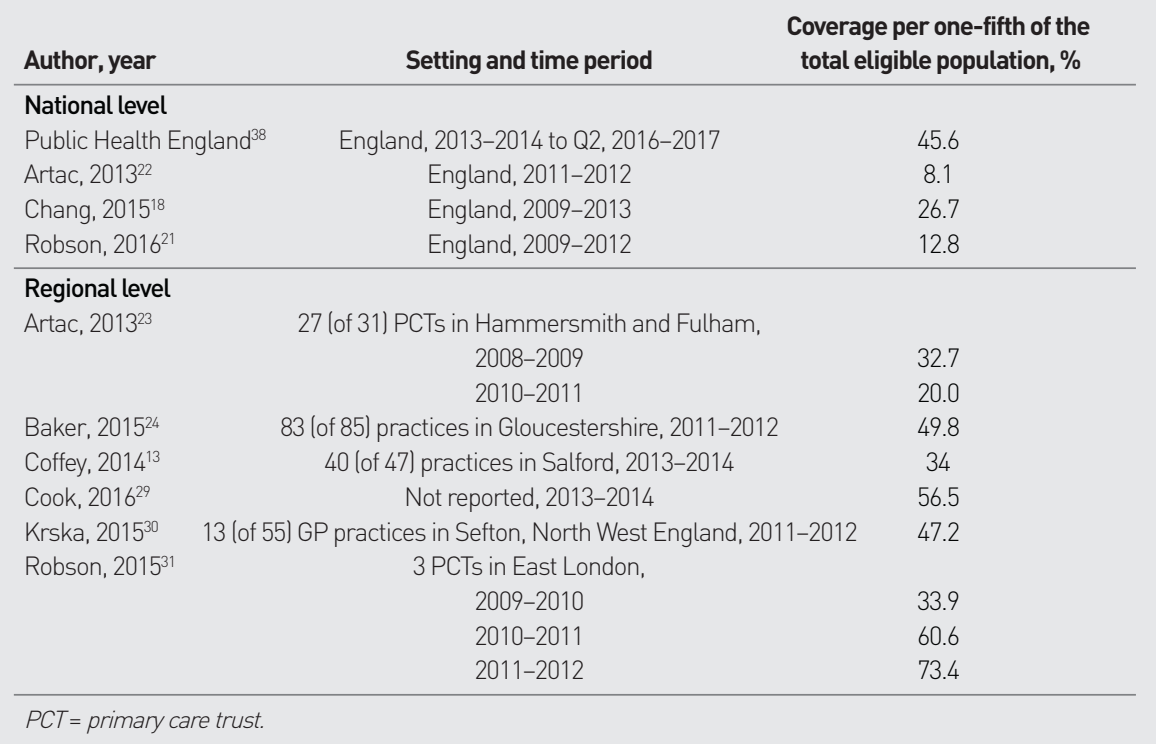

collected in particular geographic areas. ${ }^{12,16}$ Eleven studies ${ }^{15,17-23,26-28}$ were assessed as high quality (further information is available from the authors on request). In addition to the 26 included observational studies, data identified in the additional internet searches were also extracted from PHE's website. ${ }^{38}$

\section{Coverage $(n=10)$}

The PHE website included data on national level coverage during the first 3.5 years of the current 5-year cycle (2013-2014, when the NHS Health Check became a statutory requirement, to second quarter (Q2), 20162017), as well as variation in coverage over time (per quarter) and by area lat the county level). ${ }^{38}$ Nine further studies reported data on coverage (Table 1). 13,18,21-24,29-31

Reported coverage. The PHE website reported coverage of $45.6 \%$ for the whole of England (2013-2014 to Q2, 2016-2017), ranging from $18.9 \%$ in Surrey to $109.2 \%$ in Newham. ${ }^{38}$ Where full-year data were available, national coverage varied between $48.1 \%$ in $2014-2015$ to $45.0 \%$ in $2015-2016$.

Three of the nine published studies used national-level data from earlier years. $18,21,22$ The reported coverage ranged from $8.1 \%$ $(2011-2012)^{22}$ to $26.7 \%(2009-2013) .{ }^{18}$ The other six studies reported data from samples of general practices, with coverage ranging from 20.0\% (2010-2011 in Hammersmith and Fulham) ${ }^{23}$ to $73.4 \%$ (2011-2012 in East London) $)^{31}$ (Table 1).

Variation in coverage. Three studies used multiple regression to identify factors associated with differences in coverage between population groups. ${ }^{18,22,23}$ The findings from these are summarised in Table 2. Two used patient-level data. Both showed higher coverage among older people and those with a family history of coronary heart disease (CHD). The study by Artac et al additionally reported higher coverage among non-smokers, those in the most deprived tertile, those without CVD comorbidities, those registered with larger general practices, and among people from black and South Asian ethnic groups. ${ }^{23}$ By contrast, the study by Chang et al found no significant association between coverage and deprivation, and a lower coverage among people from black African and other black ethnic groups. ${ }^{18}$ The third study used data from 151 primary care trusts (PCT), and found those in the most deprived tertile were significantly more likely to have attended a health check, but no significant associations for age, ethnicity, population size, and other PCT-level measures. ${ }^{22}$

A further five studies reported coverage for different population subgroups without adjustment for covariates. 18,21,23,29,30 The two that used data from large datasets with nationwide reach during the programme's first 4 years showed higher coverage among females, older people, and those living in more deprived areas. ${ }^{18,21}$

\section{Uptake ( $n=12$ )}

The PHE website included data on national-level uptake (2013-2014 to Q2, 2016-2017), as well as variation in uptake over time (per quarter) and by area lat the county levell. Eleven studies reported uptake and socioeconomic factors associated with uptake in general practices $(n=9)^{14,26,27,29,30,32-35}$ and community-based settings $(n=2) .^{12,16}$ The study samples were different from those used in the coverage studies and generally smaller, ranging from two ${ }^{32}$ to $40^{33}$ general practices, incorporating between $1380^{34}$ and $50485^{29}$ patients.

Reported uptake. Table 3 shows the reported uptake across the data sources. The PHE website reported uptake of $48.2 \%$ for the whole of England (2013-2014 to Q2, 2016-2017), ranging from $20.1 \%$ in East Riding of Yorkshire to $100 \%$ in Leicester. Where full-year data were available, national uptake varied between $47.9 \%$ in 2015-2016 to 49.0\% in 2013-2014. Uptake in the general practice studies $(n=9)$ ranged from 27\% (four practices in the East of England $)^{34}$ to $52.9 \%$ (13 practices in North West England). ${ }^{30}$ Uptake in the community settings was $45.9 \%$ (a football ground) ${ }^{16}$ and 71.8\% (a mental healthcare unit). ${ }^{12}$ 


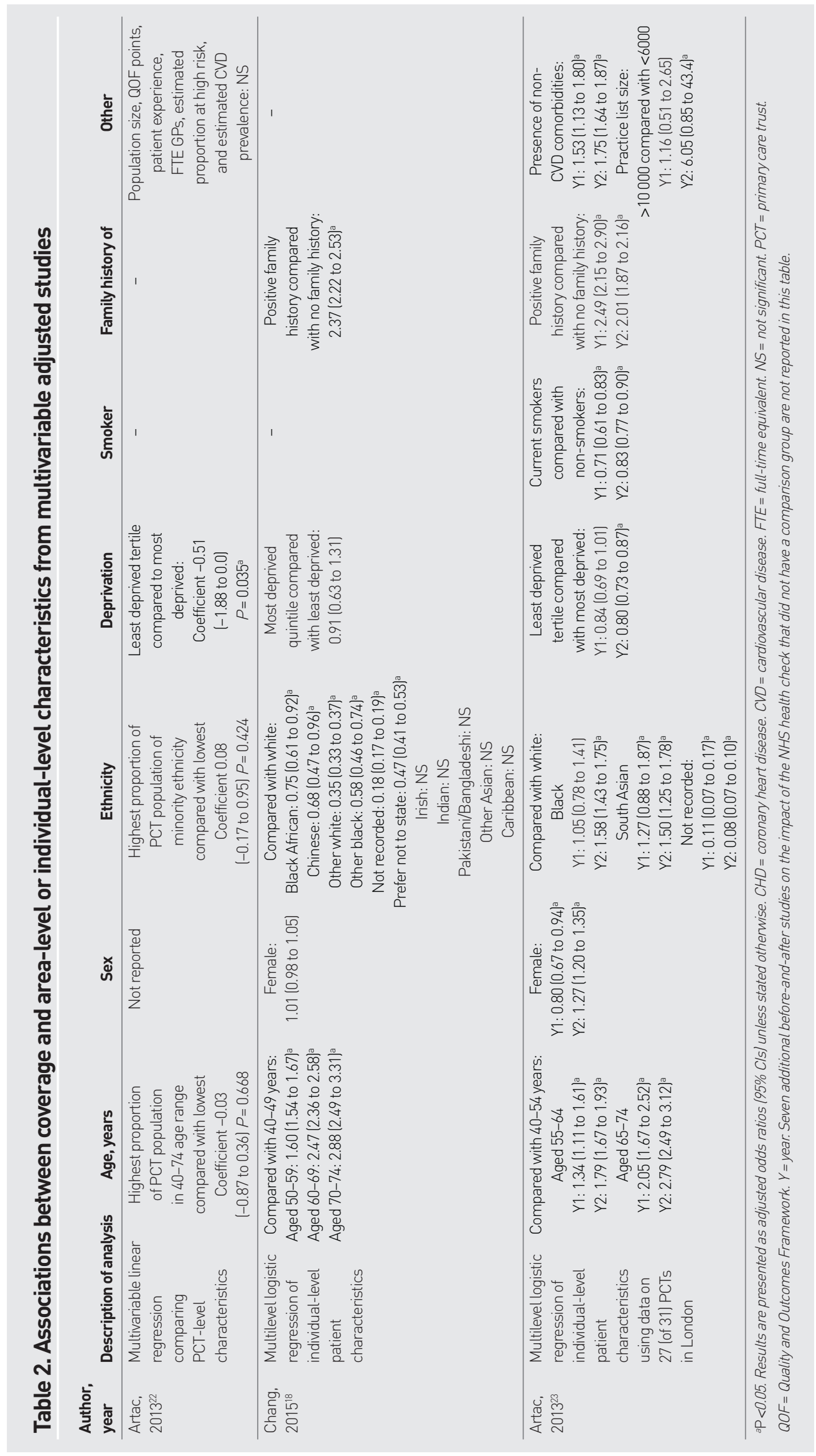


Variation in uptake. Five studies reported associations between patient characteristics and the likelihood of attending, using multivariable regression (Table 3). 14,26,27,34,35 These consistently showed that the odds of taking up an invitation increased significantly with age and lower deprivation. Of the five studies reporting associations between uptake and sex, four also showed females were more likely to take up invitations. 14,26,34,35 The fifth, a study of 37 practices in Stoke-onTrent, ${ }^{27}$ reported the opposite, with males more likely to take up invitations. Only two studies reported the effects of ethnicity. One was in 29 practices in Ealing (West London), and found invitees of South Asian or mixed ethnicity were more likely to attend than white British, while there was no difference for black or other groups, and those with missing data were less likely to attend. ${ }^{26}$ The other was across four general practices in the East of England and found no difference in uptake between participants of white and non-white ethnicity. ${ }^{34}$

Five studies also reported unadjusted comparisons between invited attendees and non-attendees. ${ }^{26,27,29,30,34}$ All reported higher uptake in older people, but findings for deprivation were more mixed, with two reporting higher uptake in those in the least deprived areas, ${ }^{27,29}$ one with higher uptake in the most deprived, ${ }^{34}$ and two with no significant differences. ${ }^{26,30}$ Notably, the association between deprivation and uptake in the unadjusted analysis of the study across four general practices in the East of England was in the opposite direction from the multivariable analysis, which adjusted for GP practice lgreater deprivation was associated with a higher odds of attending in unadjusted analysis in the study). As the authors of that study note, ${ }^{34}$ the GP practices had different distributions of deprivation and used different invitation methods, highlighting the importance of GP surgery characteristics when assessing uptake. Two studies also reported higher uptake in women ${ }^{29,34}$ and, where reported, uptake was higher in non-smokers, those with higher CVD risk, and those with hypertension or raised cholesterol. 26,27,30

\section{Impact ( $n=12$ )}

In all, 12 studies reported evidence on shortterm impact. Five included a comparison group (Table 4). Of these, two used CPRD data to examine individual-level differences over time between matched attendees and non-attendees. ${ }^{19,20}$ The other three reported population-level associations between coverage and outcome. ${ }^{15,28,36}$ The remaining seven studies were before-and-after studies without comparison groups. ${ }^{17,18,21,25,26,30,37}$ No studies of long-term health impacts or economic evaluations were identified.

Disease detection ( $\mathrm{n}=4$ ). The CPRD study by Chang et al showed more frequent diagnosis of familial hypercholesterolaemia, hypertension, CKD, peripheral vascular disease, and T2DM among attendees compared with non-attendees during the 2 years following attendance, while stroke diagnosis was significantly less likely. ${ }^{20}$ No significant differences in diagnoses of atrial fibrillation (AF), coronary artery disease, heart failure, or transient ischaemic attack were observed. ${ }^{20}$ The CPRD study by Forster et al also showed more frequent diagnosis of hypercholesterolaemia (high cholesterol), and of hypertension among males (but not females). ${ }^{19}$

Two further studies used small samples of general practices and reported associations between NHS Health Check coverage and disease detection after controlling for arealevel characteristics (for example, age profile and deprivation). ${ }^{28,36}$ The study by Caley et a ${ }^{28}$ identified no statistically significant associations between coverage and change in the prevalence of T2DM, hypertension, CHD, CKD, or AF. However, the study only included 79 general practices, and only $13.6 \%$ of the eligible population had received an NHS Health Check so it was underpowered to detect small differences. The study by Lambert et $a^{36}$ reported that the number of NHS Health Checks performed explained between $6 \%$ and $60 \%$ of the variance in incident hypertension across the different practices.

Health-related behaviour ( $\mathrm{n}=4)$. The only study with a comparison group to report health-related behaviour reported no significant association between change in smoking prevalence /recorded within primary care records over a median of 2 years) and attendance at a health check. ${ }^{20}$ Three studies reported change in smoking among individuals after attendance at a health check. Two ${ }^{17,37}$ showed a significant reduction of at least 10 percentage points in the proportion of attendees who smoked, whereas in the other the change was not statistically significant. ${ }^{25}$ However, without a comparison group it is not possible to attribute these changes to the NHS Health Check. No other health-related behaviours were reported.

Prescribing ( $\mathrm{n}=9)$. The two CPRD studies $^{19,20}$ identified significantly greater increases in statin and antihypertensive 


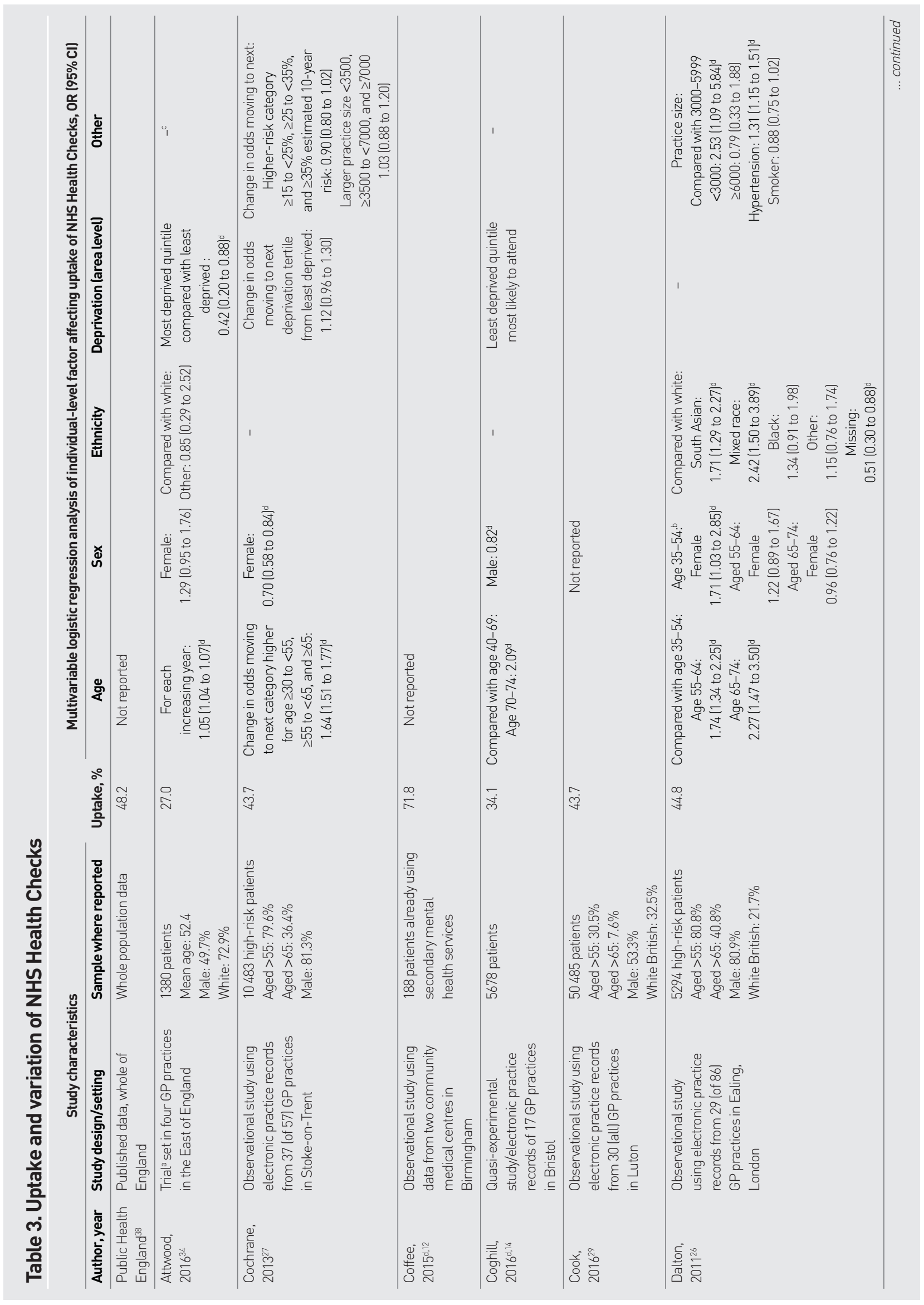




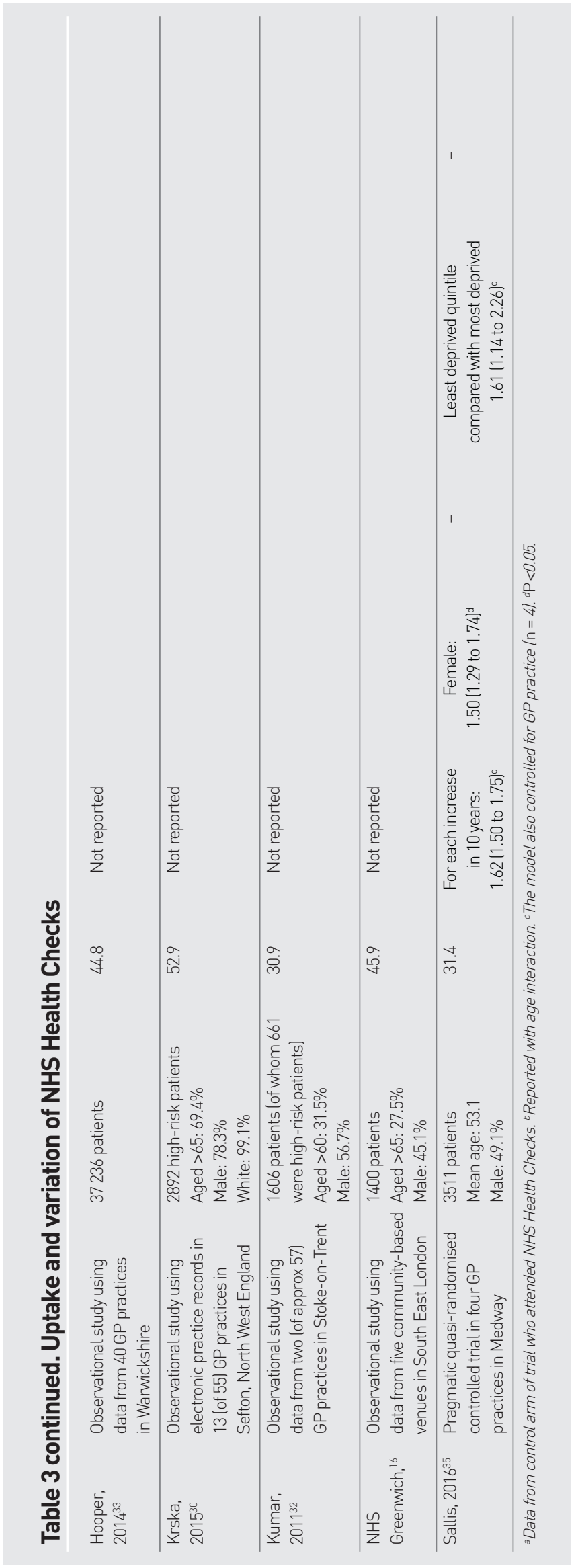

prescriptions among attendees than matched non-attendees. For example, new statin prescriptions were initiated for $5.6 \%$ of attendees, versus $1.2 \%$ of non-attendees over a median of 2 years in one of the studies, ${ }^{20}$ and by $11.0 \%$ and $7.6 \%$ over 4 years in the other..$^{19}$ Another study investigated national-level prescribing data and showed a significant association between coverage and high-dose statin prescribing at the PCT level in 2011; however, the association was not significant for low-dose statins. ${ }^{15}$

All of the six before-and-after studies showed an increased likelihood of a statin prescription following attendance. ${ }^{17,18,21,25,26,30}$ The proportion prescribed statins after the health check ranged from $18.3 \%$ in one of the CPRD studies ${ }^{17}$ to $49.9 \%$ in Hammersmith and Fulham. ${ }^{25}$

Individual risk factors and CVD risk $(n=5)$. The CPRD study by Chang et a ${ }^{20}$ showed significant differences in body mass index (BMI), blood pressure (BP, systolic and diastolic), modelled CVD risk, and total cholesterol between attendees and matched non-attendees during a 2-year period. ${ }^{20}$ For example, the QRISK2 mean score (\% 10-year risk) fell by 0.21 ( $95 \%$ confidence interval $[\mathrm{Cl}]=0.19$ to 0.24 ), from 5.1 to 4.9 among non-attendees, compared with 6.7 to 6.2 among attendees, which is equivalent to the prevention of one cardiovascular event per 4762 attendees. However, the sample used in the analysis was limited by missing data: only $2.3 \%$ of non-attendees had a follow-up QRISK2 score recorded. The population-level cross-sectional study by Lambert et al also reported a strong negative association between the number of health checks provided in a particular area and incident cases of CVD. ${ }^{36}$

Three further before-and-after studies of attendees ${ }^{17,25,37}$ identified significant reductions in diastolic BP and cholesterol levels after 12-15 months. Significant reductions in CVD risk, ${ }^{25,37}$ systolic $\mathrm{BP},{ }^{17,37}$ and some (although not all) obesity-related measures ${ }^{17,37}$ were also reported in two of the three studies. However, in addition to having no comparison group, the samples used in the analyses were also limited by missing data lfor example, follow-up data were unavailable for $50 \%$ of attendees in one study). ${ }^{37}$

\section{DISCUSSION}

\section{Summary}

In the current 5-year cycle starting in 2013, the most recent available evidence shows that $45.6 \%$ of eligible adults across England have attended an NHS Health Check. This 


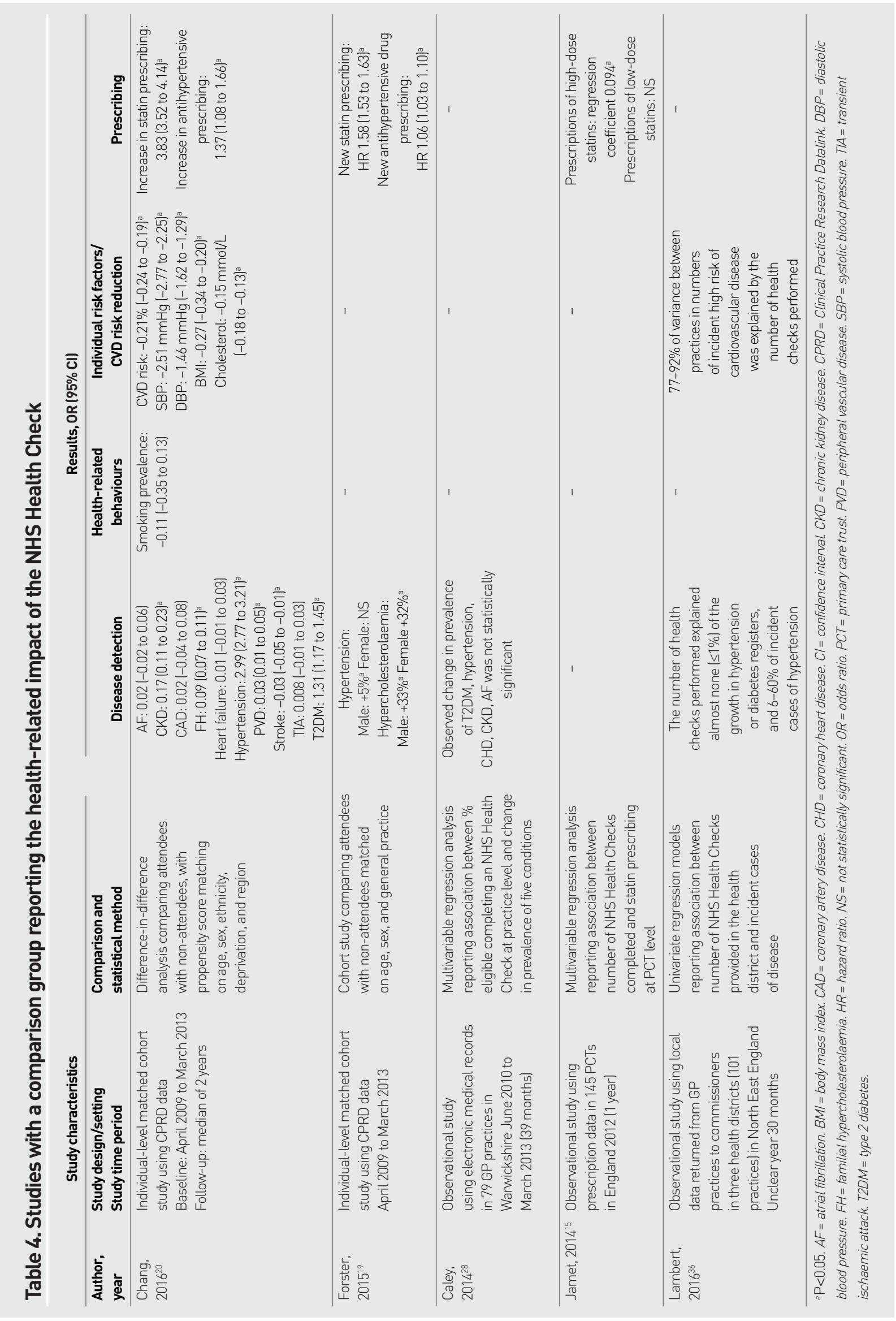


percentage varies substantially across the country, from $18.9 \%$ in some areas to $>100 \%$ in others. Data from the identified studies show higher coverage among older people, those with a family history of CHD, those living in the most deprived areas, and some ethnic groups. Uptake also varies substantially, with just under half (48.2\%) of all those invited taking up the invitation. In the selected samples of patients and general practices in the identified studies, the proportion accepting the invitation is also higher in older people and females but - in contrast to coverage - the results are lower for those living in the most deprived areas. The impact studies comparing attendees with matched non-attendees showed that attendance is associated with small increases in disease detection above routine practice, an increased likelihood of statin and antihypertensive prescribing (with the percentage of those with a modelled 10-year CVD risk $\geq 20 \%$ who were prescribed statins following a health check ranging from $18 \%$ to $63 \%$ ), and small decreases in modelled CVD risk (the best current evidence suggests that one cardiovascular event is prevented per 4762 attendees, equating to $>1400$ events across the country during a 5 -year cycle). Very few studies have reported the impact of attendance on health-related behaviours.

\section{Strengths and limitations}

Almost a decade since the programme was introduced, and 5 years since it became a statutory responsibility of local authorities, this is the first synthesis of quantitative evidence related to delivery or impact. The systematic searches, including the OpenGrey database and additional internetbased searches, are a strength of this study. However, in the absence of randomised trials or a step-wedge evaluation of a gradual rollout of NHS Health Checks, the synthesis is limited by the quality of the included studies. Studies used different populations, time points lincluding before the programme become statutory in 2013), databases, methods for identifying attendance, and (where multivariable regression was used) adjusted for different observable patient and general practice characteristics. Even for studies using electronic health records, coding was not reliable and so led to some researchers using combinations of entries to classify attendance..$^{20}$ This precluded the pooling of data from different studies. Although some studies, including the multivariable analyses of uptake (Table 3), relied on relatively small samples of general practices and patients, even the larger consolidated databases did not include nationally representative samples of patients or general practices. For example, general practices in the north of England are poorly represented in CPRD, and those that contribute data are larger $^{39}$ and potentially more engaged with research and preventive medicine than those that do not. Almost all studies relied on routinely collected data for patient characteristics and health outcomes. Missing outcome data are therefore a particular problem, as data are likely to be less complete in those people who have not attended a health check. This may be the reason why those who have attended are more likely to have a family history of $\mathrm{CHD}$ recorded, for example. There may also be systematic differences in those who attend health checks and those who do not, leading to bias in the estimates of the impact of the programme based on studies with control groups. For example, those who have not attended a health check but do have a disease or risk factor recorded may be those in whom healthcare professionals have already clinically suspected disease, or those who consult more often.

\section{Implications for research and practice}

This study identified data showing that both the anticipated coverage and uptake used in the Department of Health model were too optimistic. When judged against the (ambitious) objective of inviting all eligible individuals in each 5-year cycle, and the expected aggregate gains in population health arising from high coverage lexpected in the model to be $75 \%$ ), the evidence shows the programme has fallen considerably short. Since this remains the objective, ${ }^{2}$ a question needs to be addressed about where the necessary resources and capacity should come from to achieve it. Conversely, when judged against any reasonable valuefor-money criteria, the identified evidence on attendance is not sufficient to indicate a lack of cost-effectiveness. In the economic models, lower than anticipated coverage, for example, would merely reduce aggregated costs and aggregated health gains, without affecting the cost per QALY estimates.7,40 Like other interventions (bariatric surgery, for instance $)^{41}$ and some pharmaceuticals (which might be subjected to a budget impact test'), ${ }^{42,43}$ it seems NHS Health Checks may thus be simultaneously costeffective and unaffordable. ${ }^{40} \mathrm{~A}$ pragmatic response might be to focus attention on targeting the distribution of NHS Health Checks towards those who would benefit most, and/or towards reducing health 


\section{Funding}

This work was funded by a grant from Public Health England. Juliet Usher-Smith was funded by a National Institute for Health Research (NIHR) Clinical Lectureship and Fiona Walter by an NIHR Clinician Scientist award. Juliet Usher-Smith is now funded by a Cancer Research UK Prevention Fellowship (C55650/A21464). The views expressed in this publication are those of the authors and not necessarily those of the NHS, the NIHR, or the Department of Health.

\section{Ethical approval \\ Not applicable.}

\section{Provenance}

Freely submitted; externally peer reviewed.

\section{Competing interests}

The authors have declared no competing interests.

\section{Acknowledgements}

The authors thank patient and public representatives Kathryn Lawrence and Chris Robertson for providing helpful comments on the findings, and the NHS Health Checks Expert Scientific and Clinical Advisory Panel working group for providing them with the initial literature search conducted by Public Health England.

\section{Discuss this article}

Contribute and read comments about this article: bjgp.org/letters

inequalities. The finding that coverage (the proportion of the eligible population who have attended an NHS Health Check) among those in the most deprived areas was higher than average, despite uptake (the proportion of those invited who have attended an NHS Health Check) among those groups being lower land the findings from the study by Attwood et al in which the direction of association between sociodemographic characteristics and uptake was reversed after adjusting for GP practice), ${ }^{34}$ suggests that this is already happening to some degree. Together with the finding that coverage was higher among older people, who will be at higher risk of CVD than younger people, this may go some way towards alleviating concerns among health professionals that attendees are predominantly the 'worried well' or those least likely to benefit. ${ }^{4}$ However, given that much of the data on coverage and uptake were from different sources, the authors suggest that this should be the focus of future research. This could be supported, to some degree, through development of a slightly broader PHE dataset for the routine collection of a small number of variables on those invited and those who subsequently attend. In future years, it will also be important to distinguish between those attending for the first time and those attending follow-up NHS Health Checks after 5 years.

Although this study also showed statin prescribing to be below expectations, potentially increasing the cost per QALY, there remains a significant shortage of data on the health impacts, particularly longer term, and costs of health checks. Alongside the data on attendance identified in this study, such data are necessary for revising key assumptions in economic models of health checks, ${ }^{45,46}$ not only in England, but potentially also internationally, where similar data are also currently limited.,57 There is also a need for further high-quality studies comparing matched attendees and non-attendees, including follow-up studies to quantify the impact of health check attendance on physical activity, diet, alcohol consumption, smoking, and potential harms such as false reassurance and anxiety, which are currently unknown. 


\section{REFERENCES}

1. Department of Heath. Announcement of health checks. 2009. http://webarchive. nationalarchives.gov.uk/+/uww.dh.gov.uk/en/Healthcare/Longtermconditions/ Vascular/NHShealthcheck/index.htm (accessed 22 May 2018).

2. Public Health England. NHS Health Checks: applying All Our Health. 2018. https:// unw.gov.uk/government/publications/nhs-health-checks-applying-all-our-health/ nhs-health-checks-applying-all-our-health (accessed 22 May 2018).

3. Perk J, De Backer G, Gohlke H, et al. European guidelines on cardiovascular disease prevention in clinical practice (version 2012): the Fifth Joint Task Force of the European Society of Cardiology and Other Societies on Cardiovascular Disease Prevention in Clinical Practice (constituted by representatives of nine societies and by invited experts). Atherosclerosis 2012; 223(1): 1-68.

4. World Health Organization. Package of essential noncommunicable (PEN) disease interventions for primary health care in low-resource settings. 2010. http://apps. who.int/iris/bitstream/10665/44260/1/9789241598996 eng.pdf laccessed 22 May 2018).

5. Schuetz CA, Alperin P, Guda S, et al. A standardized vascular disease health check in Europe: a cost-effectiveness analysis. PLoS One 2013; 8(7): e66454

6. Lee JT, Lawson KD, Wan Y, et al. Are cardiovascular disease risk assessment and management programmes cost-effective? A systematic review of the evidence. Prev Med 2017; 99: 49-57.

7. Department of Health. Economic modelling for vascular checks. A technical consultation on the work undertaken to establish the clinical and cost effectivess of the evidence base for the Department of Health's policy of vascular checks. 2008. wnw.healthcheck.nhs.uk/document.php?o=225 laccessed 22 May 2018).

8. Curtis L, Burns A. Unit costs of health and social care 2016. 2016. http://mww. pssru.ac.uk/project-pages/unit-costs/unit-costs-2016/ laccessed 22 May 2018).

9. Claxton K, Martin S, Soares M, et al. Methods for the estimation of the National Institute for Health and Care Excellence cost-effectiveness threshold. Health Technol Assess 2015; 19(14): 1-503.

10. Usher-Smith J, Harte E, MacLure C, et al. Patient experience of NHS Health Checks: a systematic review and qualitative synthesis. BMJ Open 2017; 7: e017169.

11. Critical Appraisal Skills Programme (CASP). CASP tools and checklists. http:// unw.casp-uk.net/casp-tools-checklists (accessed 22 May 2018).

12. Coffee S. Engaging mental health service users in Solihull with the NHS Health Check programme: a community pilot project. 2015. http://webarchive. nationalarchives.gov.uk/20160506191316/http://www.nhsiq.nhs.uk/media/2672806/ solihull_mh_case_study.pdf laccessed 22 May 2018).

13. Coffey M, Cooper AM, Brown TM, et al. Vascular health checks in Salford: an exploration using FARSITE data, commissioned by Salford City Council. 2014 http://usir.salford.ac.uk/35581/1/final_farsite_report.pdf (accessed 22 May 2018).

14. Coghill N, Garside L, Chappell A. Improving the uptake of NHS Health Checks in more deprived communities using 'outreach telephone calls' made by specialist health advocates from the same communities: a quantitative service evaluation. In: Public Health England NHS Health Check National Conference 2016: getting serious about prevention, 1 Mar 2016, London.

15. Jamet G, Tubeuf S, Meads D. Has the introduction of NHS Health Checks increased the prescription of statins for CVD prevention? Leeds Institute of Health Sciences Working Paper Series. Leeds: University of Leeds, 2014.

16. NHS Greenwich. Evaluation of NHS Health Check plus Community Outreach Programme in Greenwich. 2011. https://www.healthcheck.nhs.uk/document. php?=52 (accessed 22 May 2018).

17. Forster AS, Dodhia $\mathrm{H}$, Booth $\mathrm{H}$, et al. Estimating the yield of NHS Health Checks in England: a population-based cohort study. J Public Health 2015; 37(2): 234-240.

18. Chang KC, Soljak M, Lee JT, et al. Coverage of a national cardiovascular risk assessment and management programme (NHS Health Check): retrospective database study. Prev Med 2015; 78(10): 1-8.

19. Forster AS, Burgess C, Dodhia H, et al. Do health checks improve risk factor detection in primary care? Matched cohort study using electronic health records. $J$ Public Health 2015; 38(3): 552-559

20. Chang K, Lee J, Vamos E, et al. Impact of the National Health Service Health Check on cardiovascular disease risk: a difference-in-differences matching analysis. CMAJ 2016; 188(10): E228-E238.

21. Robson J, Dostal I, Sheikh A, et al. The NHS Health Check in England: an evaluation of the first 4 years. BMJ Open 2016; 6: e008840.

22. Artac M, Dalton ARH, Babu H, et al. Primary care and population factors associated with NHS Health Check coverage: a national cross-sectional study. J Public Health 2013; 35(3): 431-439.
23. Artac M, Dalton ARH, Majeed A, et al. Uptake of the NHS Health Check programme in an urban setting. Fam Pract 2013; 30(4): 426-435.

24. Baker C, Loughren EA, Crone D, Kallfa N. A process evaluation of the NHS Health Check care pathway in a primary care setting. J Public Health 2015; 37(2): 202-209.

25. Artac M, Dalton ARH, Majeed A, et al. Effectiveness of a national cardiovascular disease risk assessment program (NHS Health Check): results after one year. Prev Med 2013; 57: 129-134.

26. Dalton ARH, Bottle A, Okoro C, et al. Uptake of the NHS Health Checks programme in a deprived, culturally diverse setting: cross-sectional study. J Public Health (Oxf) 2011; 33(3): 422-429

27. Cochrane T, Gidlow CJ, Kumar J, et al. Cross-sectional review of the response and treatment uptake from the NHS Health Checks programme in Stoke-on-Trent. $J$ Public Health 2013; 35(1): 92-98.

28. Caley M, Chohan P, Hooper J, Wright N. The impact of NHS Health Checks on the prevalence of disease in general practices: a controlled study. Br J Gen Pract 2014; DOI: https://doi.org/10.3399/bjgp14X681013.

29. Cook EJ, Sharp C, Randhawa G, et al. Who uses NHS health checks? Investigating the impact of ethnicity and gender and method of invitation on uptake of NHS health checks. Int J Equity Health 2016; 15: 13.

30. Krska J, du Plessis R, Chellaswamy H. Implementation of NHS Health Checks in general practice: variation in delivery between practices and practitioners. Prim Health Care Res Dev 2015; 17(4): 385-392.

31. Robson J, Dostal I, Madurasinghe V, et al. The NHS Health Check programme: implementation in east London 2009-2011. BMJ Open 2015; 5: e007578.

32. Kumar J, Chambers R, Mawby Y, et al. Delivering more with less? Making the NHS Health Check work in financially hard times: real time learning from Stokeon-Trent. Qual Prim Care 2011; 19(3): 193-199.

33. Hooper J, Chohan P, Caley M. Case detection of disease by NHS Health Checks in Warwickshire, England and comparison with predicted performance. Public Health 2014; 128(5): 475-477.

34. Attwood S, Morton K, Sutton S. Exploring equity in uptake of the NHS Health Check and a nested physical activity intervention trial. J Public Health 2016; 38(3) 560-568.

35. Sallis A, Bunten A, Bonus A, et al. The effectiveness of an enhanced invitation letter on uptake of National Health Service Health Checks in primary care: a pragmatic quasi-randomised controlled trial. BMC Fam Pract 2016; 17: 35

36. Lambert MF. Assessing potential local routine monitoring indicators of reach for the NHS Health Checks programme. Public Health 2016; 131: 92-98.

37. Cochrane T, Davey R, Iqbal Z, et al. NHS Health Checks through general practice: randomised trial of population cardiovascular risk reduction. BMC Public Health 2012; 12: 944 .

38. Public Health England. Data on NHS Health Checks. http://fingertips.phe.org.uk/ profile/nhs-health-check-detailed/data\#page/0 laccessed 22 May 2018).

39. Campbell J, Dedman D, Eaton S, et al. Is the CPRD GOLD population comparable to the UK population? Pharmacoepidemiol Drug Saf2013; 22(S1): 280.

40. Hinde S, Bojke L, Richardson G, et al. The cost-effectiveness of population health checks: have the NHS Health Checks been unfairly maligned? J Public Health 2017; 25(4): 425-431.

41. Rubin JK, Hinrichs-Krapels S, Hesketh R, et al. Identifying barriers to appropriate use of metabolic/bariatric surgery for type 2 diabetes treatment: policy lab results. Diabetes Care 2016; 39(6): 954-963.

42. National Institute for Health and Care Excellence. Budget impact test. 2017. https://www.nice.org.uk/about/what-we-do/our-programmes/nice-guidance/nicetechnology-appraisal-guidance/budget-impact-test laccessed 22 May 2018).

43. Charlton V, Littlejohns P, Kieslich K, et al. Cost effective but unaffordable: an emerging challenge for health systems. BMJ 2017; 356: 11402.

44. Mills K, Harte E, Martin A, et al. Views of commissioners, managers and healthcare professionals on the NHS Health Check programme: a systematic review. BMJ Open 2017; 7: e018606

45. Crossan C, Lord J, Ryan R, et al. Cost effectiveness of case-finding strategies for primary prevention of cardiovascular disease: a modelling study. Br J Gen Pract 2017; DOI: https://doi.org/10.3399/bjgp16X687973.

46. Kypridemos C, Allen K, Hickey GL, et al. Cardiovascular screening to reduce the burden from cardiovascular disease: microsimulation study to quantify policy options. BMJ 2016; 353: i2793.

47. Lee JT, Lawson KD, Wan Y, et al. Are cardiovascular disease risk assessment and management programmes cost effective? A systematic review of the evidence. Prev Med 2017; 99: 49-57. 